\title{
Glimpses on the ethnomedicinal plant diversity in Pindari Valley, Uttarakhand
}

\author{
Ravindra Kumar ${ }^{1,2}$, Poushali Dey ${ }^{2}$ and Priyanka Agnihotri ${ }^{1,2,3}$ \\ ${ }^{1}$ Academy of Scientific and Innovative Research (AcSIR), Kamla Nehru Nagar, Ghaziabad - 201002, India \\ ${ }^{2}$ Plant diversity, Systematics \& Herbarium Division, CSIR-National Botanical Research Institute, Rana \\ Pratap Marg, Lucknow - 226001, India \\ ${ }^{3}$ Corresponding author; e-mail: priyagni_2006@yahoo.co.in
}

[Received 07.12.2020; Revised 18.12.2020; Accepted 22.12.2020; Published 31.12.2020]

\begin{abstract}
The present study aims to document the ethno-medicinal plants from the Pindari Valley situated in the Bageshwar district of Uttarakhand. A total of 80 ethno-medicinal plants belonging to 34 families have been enlisted here. Correct nomenclature, local names, families and habit have been assigned to all the species. Herbs $(56 \%)$ form the major portion of these species followed by trees $(15 \%)$, shrubs $(13 \%)$ and climbers $(6 \%)$. Native people frequently use leaves $(23 \%)$ of the plants followed by roots $(20 \%)$, fruits $(14 \%)$, whole plants $(12 \%)$, seeds $(8 \%)$, flowers $(5 \%)$, bark $(5 \%)$ and resin/latex/oil (1\%). Asteraceae family with 10 members is the dominant family for ethnobotanically important plants, followed by Rosaceae (7) and Lamiaceae (6). All the collected plant species are found to be used by local people to treat about 55 diseases including cough, fever, jaundice, arthritis, asthma, diarrhoea, etc.
\end{abstract}

Key words: Pindari valley, ethnobotanical plants, Western Himalaya, disease.

\section{INTRODUCTION}

India, one of the mega-biodiverse countries, harbours about $7-8 \%$ of recorded species in terms of plant biodiversity in the world (Agnihotri et al. 2016). A diverse range of geographical, climatic and topographic features have resulted in a number of variety of habitats and ecosystems in the subcontinent which augment its biodiversity (Balachandhran \& Arora 2006). Presence of snow-laden Himalayas in the north, peninsula in the south, deserts in the west and wet marshy deltas in the east adds to the diversity of the country both in terms of flora and fauna.

The Himalayas, which are the youngest, highest and largest mountain range in the world foster an enormous diversity of plants and relation between plants and human beings dates back to pre-historic era. Since time immemorial, human beings are dependent on natural resources, especially plants, for fulfilling their basic needs. Especially, in mountainous region, nature furnished with rich biodiversity provides shelter and facilities for individuals who use their conventional ecological expertise to make use of local natural resources. People in these regions depend on available plants for their primary needs and thus have acquired traditional knowledge of application of these plants and their products in their day-to-day life such as food, fodder for livestock and medicinal benefits. Local people transfer this traditional knowledge to their generations using various methods like oral dialogue, folklore, written scripts, taboos etc. and therefore, it keeps on gradual expansion.

Ethnobotany deals with the investigation of plants by native people of a region for exploring their indigenous uses, owing to the intrinsic value of plants and implication towards their conservation (Pandey \& Tripathi 2017). Ethnobotanical surveys form the dynamic relationship between plants and ethnic communities or tribal people (Balick \& Cox 1996; Ford 
et al. 1994). Apart from food, fodder and shelter, utilization of plants as medicines is of considerable significance. The importance of medicinal plants in traditional health maintenance provides hints for new research areas and biodiversity conservation. Since time immemorial, medicinal plants have been the subject of man's interest, almost every society has a medicinal plant usage past. Since, there is still insufficient knowledge of the uses of medicinal plants from many remote areas of the Himalayas, it is important to obtain the variety of plant resources used by local tribal people for herbal remedies that are reliable.

Attributed to its geographical location, optimum climatic and topographic features, Uttarakhand is a reservoir of different life forms and presents rich biodiversity. Uttarakhand Himalaya is bestowed with number of glaciers, rivers, valleys and peaks, which glorify the state and add to its diversity. The Pindari Glacier is situated in Bageshwar district of Uttarakhand at an altitude that ranges from 1700 to 3660 meters above msl (Joshi et al. 2011). It is located in the upper reaches of Kumaon Himalayas, between $30^{\circ} 19^{\prime} \mathrm{N}-30^{\circ} 10^{\prime} \mathrm{N}$ latitude and $79^{\circ} 57^{\prime} \mathrm{E}-$ $80^{\circ} 4^{\prime} \mathrm{E}$ longitude with total area of $339.39 \mathrm{~km}^{2}$. It is eminent amongst the most easily available of all the Himalayan ice sheets and has provided considerable attention to naturalists since the last century. Pindar River originates from Pindari glacier and meets the Alakananda River at the junction of Karnaprayag in the Garhwal district. The glacial belt ranging from 4000 to 6000 meters above msl constitutes the famous Pindari Pass which opens communication between Martoli in Milam valley and Danpur.

Earlier reports reveal that attention has been paid to various aspects of ethnobotany and several studies have been conducted in other regions of Western Himalaya (Shah \& Joshi 1971; Singh \& Kumar 2000; Aitken \& Badola 2003; Pala et al. 2010; Rawat \& Vashistha 2011; Bisht et al. 2012; Siwach et al. 2013; Topwal \& Uniyal 2018) but till date, no such study has been done from Pindari valley. The region has remained unexplored since Rao (1960) conducted a botanical tour to Pindari glacier in 1957 to assess the phytodiversity. Considering the importance of the phytodiversity of the Pindari valley and lack of previous studies, an attempt has been made to assess the plant resources of the area and to interpret medicinal value of these natural resources used by local communities to treat various ailments on the basis of folklore knowledge among these communities.

\section{Study Area}

\section{MATERIALS AND METHODS}

Pindari valley inhabiting the Bageshwar district [30 $0^{\circ} 19^{\prime} \mathrm{N}-30^{\circ} 10^{\prime} \mathrm{N}$ latitude and $79^{\circ} 57^{\prime} \mathrm{E}-80$ '4'E longitude] falls under the Kumaon Himalaya of Uttarakhand state (Kandpal \& Sathyakumar 2010). With an area of about $339 \mathrm{sq} \mathrm{km}$, it lies between the Nanda Devi and Nandakot peaks and ends at an elevation of 3627 meters above msl (Kholiya et al. 2018). The valley is well known for its Pindari glacier. Pindari Glacier gives rise to Pindar River which drains the valley. There are about 14-15 towns in the valley inhabited by poor people who make their two ends meet by practicing negligible subsistence farming, dairying cattle for milk and horses for voyagers. The vegetation is affected by the climatic conditions and range of altitude prevailing there in the valley. The dominant vegetation includes Pinus, Acer, Juglans, Cupressus, Quercus and Rhododendron distributed from temperate to sub-alpine zone while alpine meadows occupy the area between Phurkia and Pindari Glacier which is moistly devoid of trees. The best kenned trekking course in Kumaun, pursued by the Pindari stream to its source on the ice sheet includes Nandakote $(6,860 \mathrm{~m})$. On the east and west of Pindari ice mass, Chhanguch $(6,322 \mathrm{~m})$ and Nandaghunti $(6,310 \mathrm{~m})$ peaks exist respectively while the Kaphni peak lies at the foot of Nandakote and Sunderdhunga ice sheets. Among them, Pindari ice sheet is about $3 \mathrm{~km}$ long and $0.25 \mathrm{~km}$ wide associated with the southwestern incline of the external dividers of Nanda Devi Sanctuary. 


\section{Field survey and data collection}

The present study was done in Pindari valley in the year 2019. A botanical trip was undertaken in order to assess the plant diversity of the area and to collect assessment data from their natural habitat. Efforts were made to find out the extent of economic potential of plant diversity. Interactions with the local people were made to know the mode of uses and their dependency on plant resources for the treatment of various ailments and for other beneficial purposes. Approximately, 200 plant species were collected, out of which, 80 species were found to be of medicinal importance and are frequently used by the local inhabitants of the Pindari valley. Further, published literature, online databases and other available information on the web was consulted for the medicinal value and uses of the plant species recorded from Pindari valley and also used by the other communities in Indian Himalayan region.
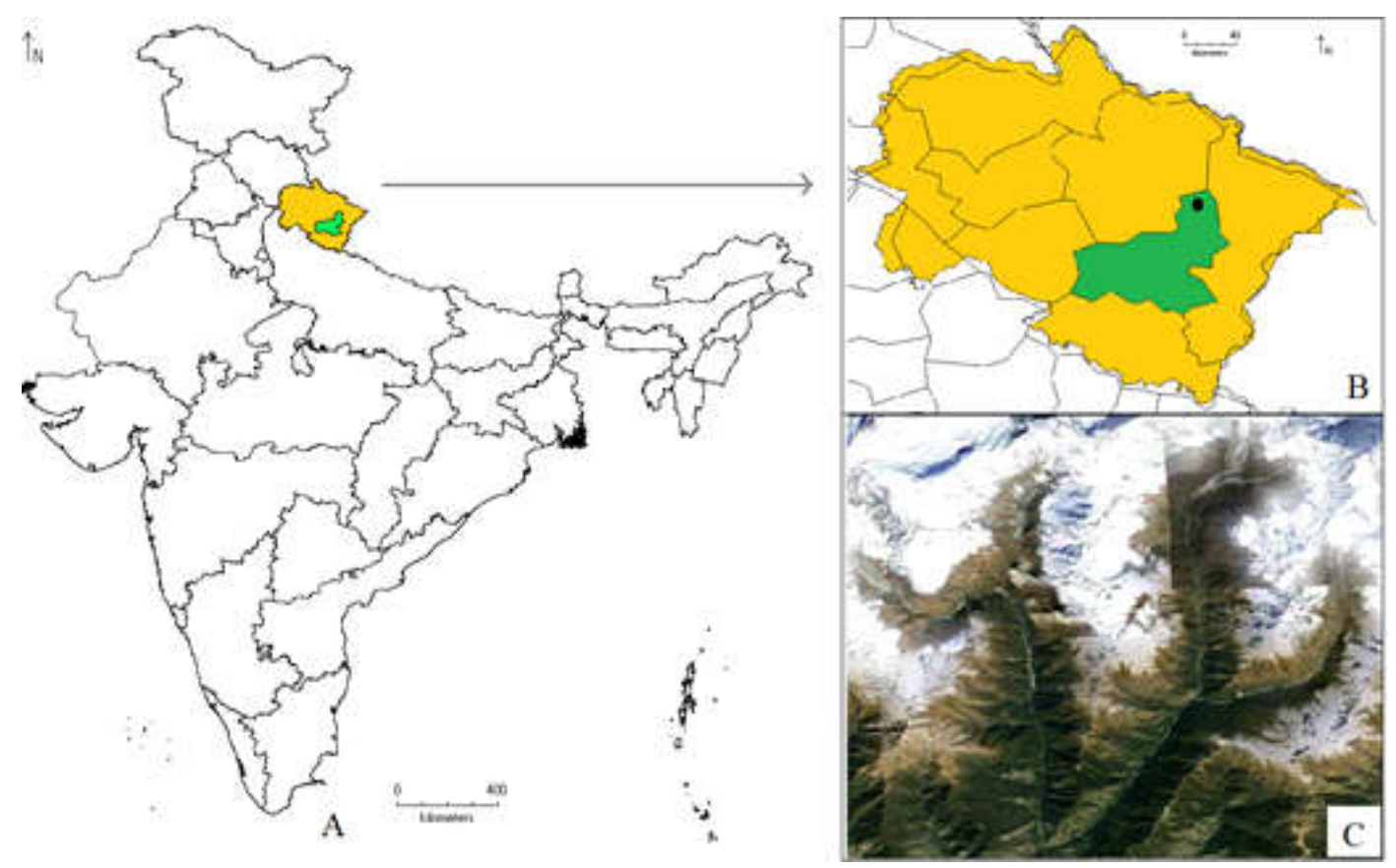

Figure 1: Map showing the position of the Pindari Valley in Uttarakhand

\section{RESULTS AND DISCUSSION}

Altogether 80 ethnobotanically important species belonging to 72 genera 34 families have been recorded from the Pindari Valley (Table 1). All the species along with their botanical names, vernacular or local names, families, parts used and uses are enumerated in Table-1. Among the recorded species 53 species were herbs, 13 were trees, 9 were shrubs 9 and 5 were climber (Figure 2). Native people mostly use leaves (30) followed by root/rhizome (26), fruit (18), whole plants (15), seeds (10), flower (7), bark (6), tuber (5), shoot/aerial parts or twigs (5), stem (2), wood (2) and oil and latex each with single species in order to cure different ailments (Figure 3). The distribution within the taxonomic families of those plants/parts is dominated by Asteraceae (10), followed by Rosaceae (7) and Lamiaceae (6), Berberidaceae, Ericaceae, Apiaceae (4 each), Amaranthaceae, Ranunculaceae (3 each), Sapindaceae, Betulaceae, Araceae, Asparagaceae, Dioscoreaceae, Caryophyllaceae, Plantaginaceae, Violaceae (2 each), Cannabaceae, Brassicaceae, Pinaceae, Solanaceae, Moraceae, Cucurbitaceae, Caprifoliaceae, Polygonaceae, 
230 Ethnomedicinal plants of Pindari valley

Table 1. List of medicinal plants recognized from the Pindari Valley

\begin{tabular}{|c|c|c|c|c|}
\hline Botanical name [Family] & Local name & $\begin{array}{l}\text { Habi } \\
t\end{array}$ & $\begin{array}{l}\text { Parts } \\
\text { used }\end{array}$ & Uses \\
\hline $\begin{array}{l}\text { Acanthophyllum cerastioides (D. Don) } \\
\text { Madhani \& Zarre [Caryophyllaceae] }\end{array}$ & & Herb & $\begin{array}{l}\text { Whole } \\
\text { plant }\end{array}$ & Boils and wounds \\
\hline $\begin{array}{l}\text { Achyranthes bidentata Blume } \\
\text { [Amaranthaceae] }\end{array}$ & Putkanda & Herb & $\begin{array}{l}\text { Whole } \\
\text { plant, } \\
\text { leaf, root }\end{array}$ & $\begin{array}{l}\text { Mouth ulcers, scorpion } \\
\text { sting, swelling, whooping } \\
\text { cough. }\end{array}$ \\
\hline $\begin{array}{l}\text { Aconitum heterophyllum Wall. ex Royle } \\
\text { [Ranunculaceae] }\end{array}$ & Atish & Herb & Root & Diarrhoea \\
\hline $\begin{array}{l}\text { Aesculus indica (Wall. ex Cambess.) Hook. } \\
\text { [Sapindaceae] }\end{array}$ & Khanor & Tree & $\begin{array}{l}\text { Seed, } \\
\text { fruit, bark }\end{array}$ & $\begin{array}{l}\text { Anthelmintic, dislocated } \\
\text { joint, diuretic, leucorrhoea } \\
\text { and veterinary } \\
\text { galactagogue }\end{array}$ \\
\hline $\begin{array}{l}\text { Ajuga integrifolia Buch.-Ham. ex D. Don } \\
\text { [Lamiaceae] }\end{array}$ & Neelkabi & Herb & Leaf & Acidity and indigestion \\
\hline Alnus nepalensis D.Don [Betulaceae] & Utish & Tree & $\begin{array}{l}\text { Latex, } \\
\text { bark }\end{array}$ & Diuretic \\
\hline $\begin{array}{l}\text { Anaphalis triplinervis Sims ex C.B. Clarke } \\
\text { [Asteraceae] }\end{array}$ & $\begin{array}{l}\text { Woolly Pearly } \\
\text { Everlasting }\end{array}$ & Herb & $\begin{array}{l}\text { Leaf, } \\
\text { whole } \\
\text { plant }\end{array}$ & Diuretic \\
\hline Angelica glauca Edgew. [Apiaceae] & Chaura & Herb & Root & $\begin{array}{l}\text { Dysentery, gastric } \\
\text { complaints, menorrhea, } \\
\text { stomach complaints, } \\
\text { vomiting }\end{array}$ \\
\hline $\begin{array}{l}\text { Arisaema intermedium Blume } \\
\text { [Araceae] }\end{array}$ & Nagdaman & Herb & $\begin{array}{l}\text { Roots, } \\
\text { Stem }\end{array}$ & $\begin{array}{l}\text { Dehydration, fever, } \\
\text { intestinal pain, body ache } \\
\text { and skin infection }\end{array}$ \\
\hline Atisaema tortuosum (Wall.) Schott [Araceae] & Nagdaman & Herb & Tuber & $\begin{array}{l}\text { Rheumatism and } \\
\text { breathing disorder }\end{array}$ \\
\hline $\begin{array}{l}\text { Artemisia parviflora Roxb. ex D.Don } \\
\text { [Asteraceae] }\end{array}$ & Jhau & Herb & $\begin{array}{l}\text { Leaf, } \\
\text { Root, } \\
\text { Seed }\end{array}$ & $\begin{array}{l}\text { Throat problems, } \\
\text { vermifuge and } \\
\text { carminative }\end{array}$ \\
\hline $\begin{array}{l}\text { Asparagus filicinus Buch.-Ham. ex D. Don } \\
\text { [Asparagaceae] }\end{array}$ & Shatavari & Herb & Root & $\begin{array}{l}\text { Antitussive, Diuretic } \\
\text { expectorant, nervine, } \\
\text { stomach ache, tonic }\end{array}$ \\
\hline Berberis aristata DC. [Berberidaceae] & Kambal & Shrub & $\begin{array}{l}\text { Stem, } \\
\text { Bark, } \\
\text { Wood, } \\
\text { Root }\end{array}$ & $\begin{array}{l}\text { Eye complaints, } \\
\text { diarrhoea, jaundice, skin } \\
\text { diseases, syphilis, chronic } \\
\text { rheumatism, urinary } \\
\text { disorders, diabetes, } \\
\text { jaundice and gastro } \\
\text { intestinal problem. }\end{array}$ \\
\hline $\begin{array}{l}\text { Berberis asiatica Roxb. ex DC. } \\
\text { [Berberidaceae] }\end{array}$ & Kilmore & Shrub & $\begin{array}{l}\text { Root, } \\
\text { Fruits }\end{array}$ & $\begin{array}{l}\text { Diaphoretic, astringent, } \\
\text { ulcers urethral, ulcers and } \\
\text { piles }\end{array}$ \\
\hline Berberis lycium Royle [Berberidaceae] & Darubaridra & Shrub & $\begin{array}{l}\text { Roots, } \\
\text { fruits }\end{array}$ & Indigestion and fever \\
\hline Betula utilis D.Don [Betulaceae] & Bhuj & Tree & $\begin{array}{l}\text { Papery } \\
\text { bark and } \\
\text { young } \\
\text { leaves }\end{array}$ & $\begin{array}{l}\text { Skin disinfectant, } \\
\text { convulsions, wound } \\
\text { healing, bronchitis and } \\
\text { leprosy }\end{array}$ \\
\hline
\end{tabular}


Ravindra Kumar et al. 231

\begin{tabular}{|c|c|c|c|c|}
\hline Botanical name [Family] & Local name & $\begin{array}{l}\text { Habi } \\
\mathrm{t}\end{array}$ & $\begin{array}{l}\text { Parts } \\
\text { used }\end{array}$ & Uses \\
\hline Brucea javanica (L.) Merr. [Simaroubaceae] & Titri & Shrub & Leaf, fruit & $\begin{array}{l}\text { Cholera, gastric } \\
\text { complaints, } \\
\text { constipation and skin } \\
\text { disease }\end{array}$ \\
\hline Caltha palustris L. [Ranunculaceae] & Shomalap & Herb & Root, leaf & Gonorrhoea \\
\hline Cannabis sativa L. [Cannabaceae] & Bhang & Herb & $\begin{array}{l}\text { Leaves } \\
\text { flower, } \\
\text { seeds }\end{array}$ & $\begin{array}{l}\text { Chronic pain, muscle } \\
\text { spasms and honey bee } \\
\text { sting }\end{array}$ \\
\hline $\begin{array}{l}\text { Capsella bursa-pastoris (L.) Medik. } \\
\text { (Brassicaceae] }\end{array}$ & Torighash & Herb & $\begin{array}{l}\text { Whole } \\
\text { plant }\end{array}$ & $\begin{array}{l}\text { Blood pressure, diarrhoea } \\
\text { and dropsy }\end{array}$ \\
\hline $\begin{array}{l}\text { Cedrus deodara (Roxb. ex D. Don) G. Don } \\
\text { [Pinaceae] }\end{array}$ & Dyar & Tree & $\begin{array}{l}\text { Oil, } \\
\text { Wood }\end{array}$ & $\begin{array}{l}\text { Anthelmintic, rheumatism } \\
\text { and ulcers }\end{array}$ \\
\hline Centella asiatica (L.) Urb. [Apiaceae] & Brabmi & Herb & Leaves & Leprosy \\
\hline Chenopodium album L. [Amaranthaceae] & Bathua & Herb & $\begin{array}{l}\text { Leaf, } \\
\text { Seed, } \\
\text { Whole } \\
\text { plant }\end{array}$ & $\begin{array}{l}\text { Revive taste, skin disease } \\
\text { and urinary complaints }\end{array}$ \\
\hline Cyathula capitata Moq. [Amaranthaceae] & $\begin{array}{l}\text { Letkural } \\
\text { Ketari }\end{array}$ & Herb & $\begin{array}{l}\text { Leaf, } \\
\text { Seed }\end{array}$ & Emetic and abortifacient \\
\hline Datura stramonium L. [Solanaceae] & Dhatura & Herb & $\begin{array}{l}\text { Leaf, } \\
\text { Fruit }\end{array}$ & $\begin{array}{l}\text { Veterinary lactation, } \\
\text { Parkinson's, asthma and } \\
\text { diarrhoea }\end{array}$ \\
\hline Dioscorea bulbifera L. [Dioscoreaceae] & Genthi & $\begin{array}{l}\text { Climb } \\
\text { er }\end{array}$ & $\begin{array}{l}\text { Tuber, } \\
\text { Leaves }\end{array}$ & $\begin{array}{l}\text { Urinary discharge, sore } \\
\text { throat, breast cancers, } \\
\text { Leucoderma and } \\
\text { bronchitis }\end{array}$ \\
\hline $\begin{array}{l}\text { Dioscorea deltoidea Wall. ex Griseb. } \\
\text { [Dioscoreaceae] }\end{array}$ & Singli-Mingli & $\begin{array}{l}\text { Climb } \\
\text { er }\end{array}$ & Root & $\begin{array}{l}\text { Contraceptive, } \\
\text { constipation and asthma }\end{array}$ \\
\hline $\begin{array}{l}\text { Drymaria cordata (L.) Willd. ex Schult. } \\
\text { [Caryophyllaceae] }\end{array}$ & Pithpapra & Herb & $\begin{array}{l}\text { Aerial } \\
\text { parts }\end{array}$ & Cold and bronchitis \\
\hline $\begin{array}{l}\text { Erigeron multiradiatus (Lindl. ex DC.) } \\
\text { Benth. \& Hook.f. [Asteraceae] }\end{array}$ & $\begin{array}{l}\text { Himalayan } \\
\text { fleabane }\end{array}$ & Herb & $\begin{array}{l}\text { Aerial } \\
\text { part }\end{array}$ & Blood purification \\
\hline $\begin{array}{l}\text { Eschenbachia stricta (Willd.) Raizada } \\
\text { [Asteraceae] }\end{array}$ & Horse weed & Herb & $\begin{array}{l}\text { Whole } \\
\text { plant }\end{array}$ & $\begin{array}{l}\text { Bone fracture and } \\
\text { swelling }\end{array}$ \\
\hline Ficus palmata Forssk. [Moraceae] & Bedu & Tree & $\begin{array}{l}\text { Fruit, } \\
\text { Twigs }\end{array}$ & $\begin{array}{l}\text { Lung and bladder diseases } \\
\text { and skin diseases }\end{array}$ \\
\hline Fragaria vesca L. [Rosaceae] & Gandkaphal & Herb & $\begin{array}{l}\text { Fruit, } \\
\text { Leaves }\end{array}$ & $\begin{array}{l}\text { Astringent, problems of } \\
\text { urinary organs diuretic } \\
\text { and diarrhoea }\end{array}$ \\
\hline Galinsoga parviflora Cav. [Asteraceae] & Banmara & Herb & Leaf & $\begin{array}{l}\text { Snakebite and stop } \\
\text { bleeding }\end{array}$ \\
\hline $\begin{array}{l}\text { Gaultheria nummularioides D.Don } \\
\text { [Ericaceae] }\end{array}$ & Jalan-thrait & Tree & $\begin{array}{l}\text { Leaf, } \\
\text { Fruit }\end{array}$ & $\begin{array}{l}\text { Antiseptic, carminative } \\
\text { and neural stimulant }\end{array}$ \\
\hline Globba racemosa Sm. [Zingiberaceae] & $\begin{array}{l}\text { Gundenoppima } \\
n d u\end{array}$ & Herb & Fruit & $\begin{array}{l}\text { Heart pain and stomach } \\
\text { pain }\end{array}$ \\
\hline Hedera nepalensis K. Koch [Araliaceae] & Katari & $\begin{array}{l}\text { Climb } \\
\text { er }\end{array}$ & $\begin{array}{l}\text { Whole } \\
\text { plant }\end{array}$ & $\begin{array}{l}\text { Stimulant, diaphoretic, } \\
\text { cathartic, rheumatism and } \\
\text { emmenagogue. }\end{array}$ \\
\hline $\begin{array}{l}\text { Jacobaea analoga (DC.) Veldkamp } \\
\text { [Asteraceae] }\end{array}$ & & Herb & $\begin{array}{l}\text { Whole } \\
\text { plant }\end{array}$ & $\begin{array}{l}\text { Inflammation of mouth } \\
\text { and sore throat }\end{array}$ \\
\hline
\end{tabular}


232 Ethnomedicinal plants of Pindari valley

\begin{tabular}{|c|c|c|c|c|}
\hline Botanical name [Family] & Local name & $\begin{array}{l}\text { Habi } \\
t\end{array}$ & $\begin{array}{l}\text { Parts } \\
\text { used }\end{array}$ & Uses \\
\hline Lamium album L. [Lamiaceae] & Banbuti & Herb & $\begin{array}{l}\text { Roots/ } \\
\text { rhizomes }\end{array}$ & Burns and cuts \\
\hline Leucas lanata Benth. [Lamiaceae] & Bis-kapra & Herb & $\begin{array}{l}\text { Whole } \\
\text { plant }\end{array}$ & Whooping cough \\
\hline $\begin{array}{l}\text { Lyonia ovalifolia (Wall.) Drude } \\
\text { (Ericaceae) }\end{array}$ & Ayaar & Shrub & Leaf & Skin diseases \\
\hline $\begin{array}{l}\text { Meconopsis aculeate Royle [Papaver- } \\
\text { aceae] }\end{array}$ & Veerbuti & Herb & $\begin{array}{l}\text { Flowers } \\
\text { and roots }\end{array}$ & Curing the eye diseases \\
\hline $\begin{array}{l}\text { Melanoseris violifolia (Decne.) N. Kilian } \\
\text { [Asteraceae] }\end{array}$ & & Herb & Seed, leaf & Menorrhoea \\
\hline $\begin{array}{l}\text { Nardostachys jatamansi (D. Don) DC. } \\
\text { [Caprifoliaceae] }\end{array}$ & Jatamansi & Herb & Root & $\begin{array}{l}\text { Rhizome as tonic, } \\
\text { stimulant, anti-spasmodic } \\
\text { and diuretic }\end{array}$ \\
\hline Ocimum tenuiflorum L. [Lamiaceae] & Tulsi & Herb & $\begin{array}{l}\text { Whole } \\
\text { plant }\end{array}$ & $\begin{array}{l}\text { Bronchitis, diaphoretic, } \\
\text { anti-periodic, gastric } \\
\text { disorders and malaria }\end{array}$ \\
\hline Origanum vulgare L. [Lamiaceae] & Maruwa & Herb & $\begin{array}{l}\text { Fresh and } \\
\text { dried } \\
\text { leaves } \\
\text { (both) }\end{array}$ & Chickenpox \\
\hline Oxyria digyna (L.) Hill [Polygonaceae] & Chyakulti & Herb & $\begin{array}{l}\text { Leaves } \\
\text { and } \\
\text { young } \\
\text { shoots }\end{array}$ & Dysentery \\
\hline Paris polyphylla Sm. [Melanthiaceae] & Bada Satuwa & Herb & $\begin{array}{l}\text { Tuber } \\
\text { and roots }\end{array}$ & $\begin{array}{l}\text { Fever, headache, burns, } \\
\text { wounds and poisoning }\end{array}$ \\
\hline $\begin{array}{l}\text { Parnassia nubicola Wall. ex Royle } \\
\text { [Celastraceae] }\end{array}$ & Phutkya & Herb & Tuber & Food poisoning \\
\hline $\begin{array}{l}\text { Parochetus communis Buch. - Ham. ex } \\
\text { D.Don [Fabaceae] }\end{array}$ & $\begin{array}{l}\text { Jangalee } \\
\text { Badaame Jhaar }\end{array}$ & Shrub & Leaf & $\begin{array}{l}\text { Stomach disease of babies } \\
\text { and earache }\end{array}$ \\
\hline $\begin{array}{l}\text { Picrorhiza kurroa Royle ex Benth. } \\
\text { [Plantaginaceae] }\end{array}$ & Kutaki & Herb & Rhizomes & $\begin{array}{l}\text { Fever, stomach ache, } \\
\text { jaundice, dysentery }\end{array}$ \\
\hline $\begin{array}{l}\text { Pimpinella acuminata (Edgew.) C.B. Clarke } \\
\text { [Apiaceae] }\end{array}$ & & Herb & $\begin{array}{l}\text { Whole } \\
\text { plant }\end{array}$ & Diarrhoea and dysentery \\
\hline Pimpinella diversifolia DC. [Apiaceae] & Bazeer & Herb & Root & $\begin{array}{l}\text { Digestive disorder, cold, } \\
\text { cough and leucorrhoea }\end{array}$ \\
\hline Plantago erosa Wall. [Plantaginaceae] & Isabagol & Herb & $\begin{array}{l}\text { Root \& } \\
\text { leaf }\end{array}$ & $\begin{array}{l}\text { Bone fracture, } \\
\text { inflammation and cooling } \\
\text { agent }\end{array}$ \\
\hline $\begin{array}{l}\text { Podophyllum hexandrum Royle } \\
\text { [Berberidaceae] }\end{array}$ & Bankakri & Herb & $\begin{array}{l}\text { Roots, } \\
\text { fruits and } \\
\text { flowers }\end{array}$ & $\begin{array}{l}\text { Headache, high fever and } \\
\text { abdominal pains }\end{array}$ \\
\hline $\begin{array}{l}\text { Polygonatum verticillatum (L.) All. } \\
\text { [Asparagaceae] }\end{array}$ & Salam Mishri & Herb & $\begin{array}{l}\text { Tuber } \\
\text { and leaf }\end{array}$ & General weakness \\
\hline $\begin{array}{l}\text { Potentilla eriocarpa Wall. ex Lehm. } \\
\text { [Rosaceae] }\end{array}$ & Flanchei & Herb & Leaves & $\begin{array}{l}\text { Diarrhoea, arthritis and } \\
\text { kidney stones }\end{array}$ \\
\hline Potentilla fulgens Wall. ex Sims [Rosaceae] & Bajradanti & Herb & Root & $\begin{array}{l}\text { Diarrhoea and teeth } \\
\text { cleaning }\end{array}$ \\
\hline Prinsepia utilis Royle [Rosaceae] & Bhekal & Shrub & $\begin{array}{l}\text { Fruit and } \\
\text { seed }\end{array}$ & Joint pain \\
\hline $\begin{array}{l}\text { Prunus cornuta (Wall. ex Royle) Steud. } \\
\text { [Rosaceae] }\end{array}$ & Jатmи & Tree & $\begin{array}{l}\text { Fruits } \\
\text { and ker- } \\
\text { nels }\end{array}$ & Joint pain \\
\hline
\end{tabular}


Ravindra Kumar et al. 233

\begin{tabular}{|c|c|c|c|c|}
\hline Botanical name [Family] & Local name & Habit & $\begin{array}{l}\text { Parts } \\
\text { used }\end{array}$ & Uses \\
\hline $\begin{array}{l}\text { Pseudognaphalium hypoleucum (DC.) } \\
\text { Hilliard \& B.L. Burtt [Asteraceae] }\end{array}$ & Dhoop & Herb & Paste & Cough and backache \\
\hline Pyrus pashia Buch.-Ham. ex D.Don [Rose & ackbed, $]$ Mehal & Tree & $\begin{array}{l}\text { Fruit, } \\
\text { bark }\end{array}$ & $\begin{array}{l}\text { Blood purification and eye } \\
\text { infection }\end{array}$ \\
\hline $\begin{array}{l}\text { Rhododendron arboreum } \mathrm{Sm} . \\
\text { [Ericaceae] }\end{array}$ & Burans & Tree & Flower & Diarrhoea and dysentery \\
\hline $\begin{array}{l}\text { Rhododendron campanulatum D.Don } \\
\text { [Ericaceae] }\end{array}$ & Simru & Tree & $\begin{array}{l}\text { Leaf, } \\
\text { flower, } \\
\text { root }\end{array}$ & Diarrhoea and dysentery. \\
\hline $\begin{array}{l}\text { Sapindus mukorossi Gaertn. } \\
\text { [Sapindaceae] }\end{array}$ & Ritha & Tree & $\begin{array}{l}\text { Fruit, } \\
\text { seed }\end{array}$ & $\begin{array}{l}\text { Epilepsy, causing vomiting, } \\
\text { dental cavity, cough and cold }\end{array}$ \\
\hline $\begin{array}{l}\text { Sarcococca saligna (D.Don) Müll.Arg. } \\
\text { [Buxaceae] }\end{array}$ & Chirbeeri & Shrub & Fruit & Joint pain \\
\hline $\begin{array}{l}\text { Saussurea obvallata (DC.) Sch.Bip. } \\
\text { [Asteraceae] }\end{array}$ & Brahmkamal & Herb & Flower & Mental disorder \\
\hline Smilax aspera L. [Smilacaceae] & Kukurdar & $\begin{array}{l}\text { Climb } \\
\text { er }\end{array}$ & $\begin{array}{l}\text { Root, } \\
\text { Shoot }\end{array}$ & $\begin{array}{l}\text { Skin eruption, sores and } \\
\text { wounds }\end{array}$ \\
\hline $\begin{array}{l}\text { Solena heterophylla Lour. } \\
\text { [Cucurbitaceae] }\end{array}$ & Blakari & $\begin{array}{l}\text { Climb } \\
\text { er }\end{array}$ & $\begin{array}{l}\text { Leaf, } \\
\text { fruit, root }\end{array}$ & $\begin{array}{l}\text { Antifertility, tooth ache cold, } \\
\text { diabetes, ear ache, fever, } \\
\text { Snakebite, and stomach pain. }\end{array}$ \\
\hline Sorbus lanata (D.Don) S.Schauer [Rosace & aefhuaei & Tree & Fruits & Antidiabetic \\
\hline $\begin{array}{l}\text { Stellaria media (L.) Vill. } \\
\text { [Caryophyllaceae] }\end{array}$ & Badyalu & Herb & $\begin{array}{l}\text { Whole } \\
\text { plant }\end{array}$ & Bone fracture \\
\hline $\begin{array}{l}\text { Swertia chirayita (Roxb.) H. Karst. } \\
\text { [Gentianaceae] }\end{array}$ & Chirayita & Herb & $\begin{array}{l}\text { Whole } \\
\text { plant }\end{array}$ & Antipyretic \\
\hline $\begin{array}{l}\text { Taraxacum officinale F.H. Wigg. } \\
\text { [Asteraceae] }\end{array}$ & Kanphul & Herb & Root, leaf & $\begin{array}{l}\text { Blisters, blood purification, } \\
\text { diuretic, dysentery, gastric } \\
\text { ulcers, headache, liver } \\
\text { complaints, wounds }\end{array}$ \\
\hline Taxus baccata L. [Taxaceae] & Thuner & Tree & $\begin{array}{l}\text { Leaf, } \\
\text { bark }\end{array}$ & $\begin{array}{l}\text { Antifungal, antibacterial and } \\
\text { antitumor }\end{array}$ \\
\hline $\begin{array}{l}\text { Thalictrum foliolosum DC. } \\
\text { [Ranunculaceae] }\end{array}$ & Mamiri & Herb & Root & $\begin{array}{l}\text { Abdominal pain, blood } \\
\text { purification, earache, eye } \\
\text { problems, fever, } \\
\text { leukoderma, piles, boils and } \\
\text { toothache }\end{array}$ \\
\hline Thymus serpyllum L. [Lamiaceae] & Banajwain & Herb & $\begin{array}{l}\text { Aerial } \\
\text { parts }\end{array}$ & $\begin{array}{l}\text { Asthma, epilepsy, whooping } \\
\text { cough, bronchitis and eye } \\
\text { problems. }\end{array}$ \\
\hline Urtica dioica L. [Urticaceae] & Kandali & Herb & Leaf & Allergy and muscular pain \\
\hline Verbascum thapsus L. [Scrophulariaceae] & Tamaku & Herb. & $\begin{array}{l}\text { Whole } \\
\text { Plant }\end{array}$ & Vomiting \\
\hline Viola biflora L. [Violaceae] & Banafsha & Herb & $\begin{array}{l}\text { Leaf, } \\
\text { flower, } \\
\text { root, } \\
\text { seed. }\end{array}$ & $\begin{array}{l}\text { Constipation, cold, cough, } \\
\text { diaphoretic, causing vomiting, } \\
\text { fever, leukoderma }\end{array}$ \\
\hline Viola canescens Wall. [Violaceae] & Banafsha & Herb & $\begin{array}{l}\text { Whole } \\
\text { plant }\end{array}$ & $\begin{array}{l}\text { Diaphoresis, epilepsy, blood } \\
\text { purification, treatment of skin } \\
\text { diseases, ear-ache. }\end{array}$ \\
\hline Zanthoxylum armatum DC. [Rutaceae] & Timur & Shrub & $\begin{array}{l}\text { Bark, } \\
\text { fruit }\end{array}$ & $\begin{array}{l}\text { Toothache treat colds, coughs, } \\
\text { stomachache }\end{array}$ \\
\hline
\end{tabular}

Araliaceae, Papaveraceae, Melanthiaceae, Celastraceae, Fabaceae, Simaroubaceae, Buxaceae, Smilacaceae, Gentianaceae, Taxaceae, Urticaceae, Scrophulariaceae, Rutaceae, Zingiberaceae (1) each) (Figure 4). A total of ca. 55 diseases are cured by these 80 plant species and the largest 
number of medicinal plants (i.e., 10) were reported to cure stomach ache, diarrhoea; followed by skin diseases (9); cough ; fever, cuts and wounds, urinary disorders (8 each); dysentery (7); bronchitis, joint pain (6 each); asthma jaundice, bone fracture, gastric (4 each); head ache, earache, leukoderma, indigestion (3 each), insect sting, ulcer, leprosy, piles, bleeding, boils, burns (2 each); itching, menstrual disorders diabetes, leucorrhoea, stone, body ache, digestive, constipation (1 each) (Dutt et al. 2014; Kumari et al. 2018; Naithani \& Nand 2018; Pandey et al. 2017; Rana \& Samant 2011; Topwal \& Uniyal 2018).

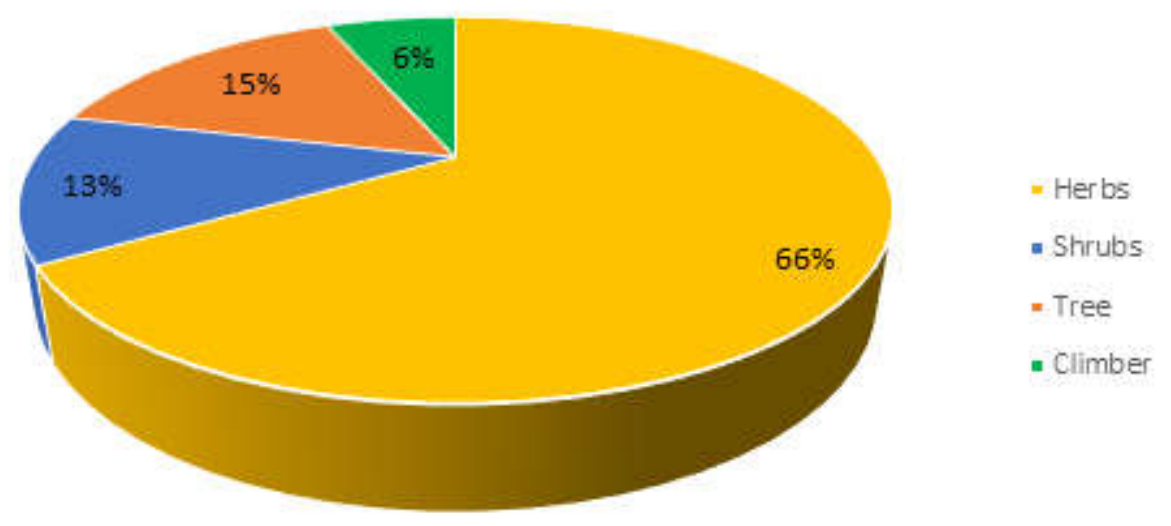

Figure 2. Habit classes of recorded ethno-medicinally important plants used in Pindari valley

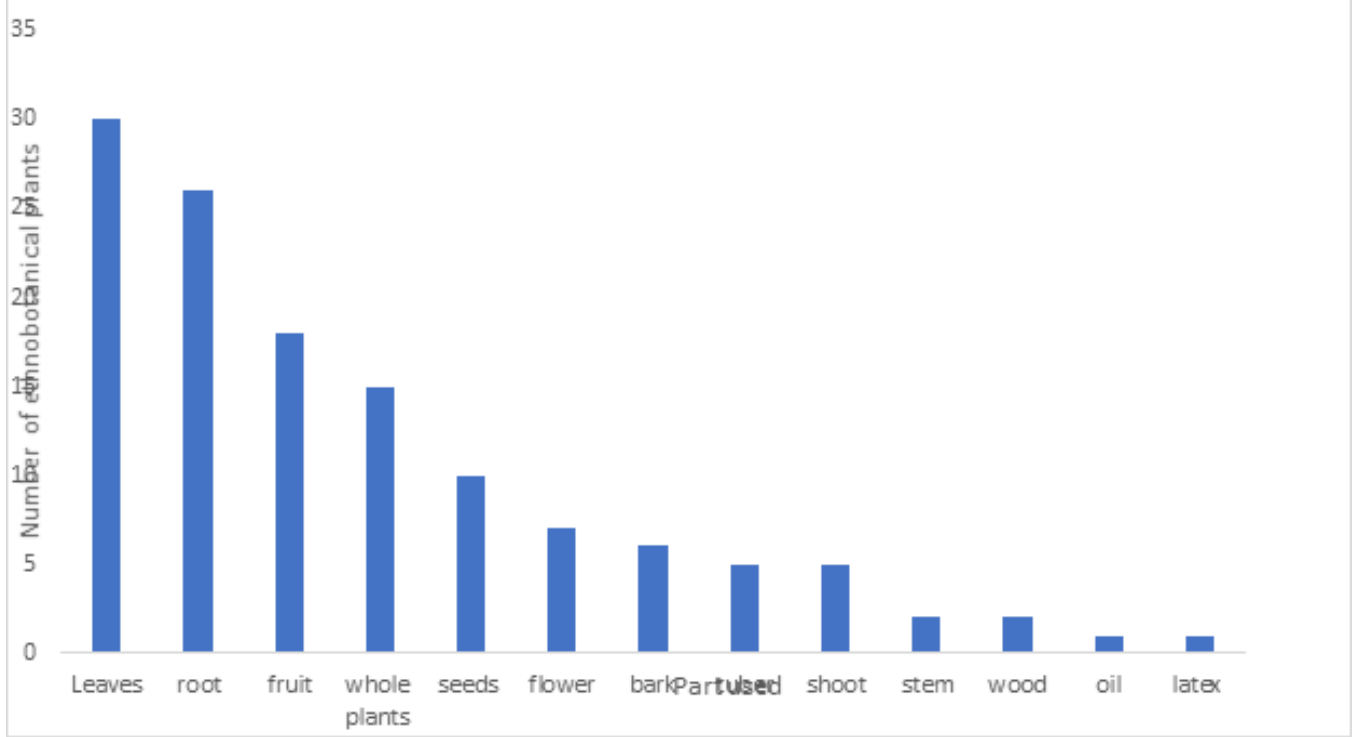

Figure 3. Contribution of different plant parts used for ethnomedicinal purpose 

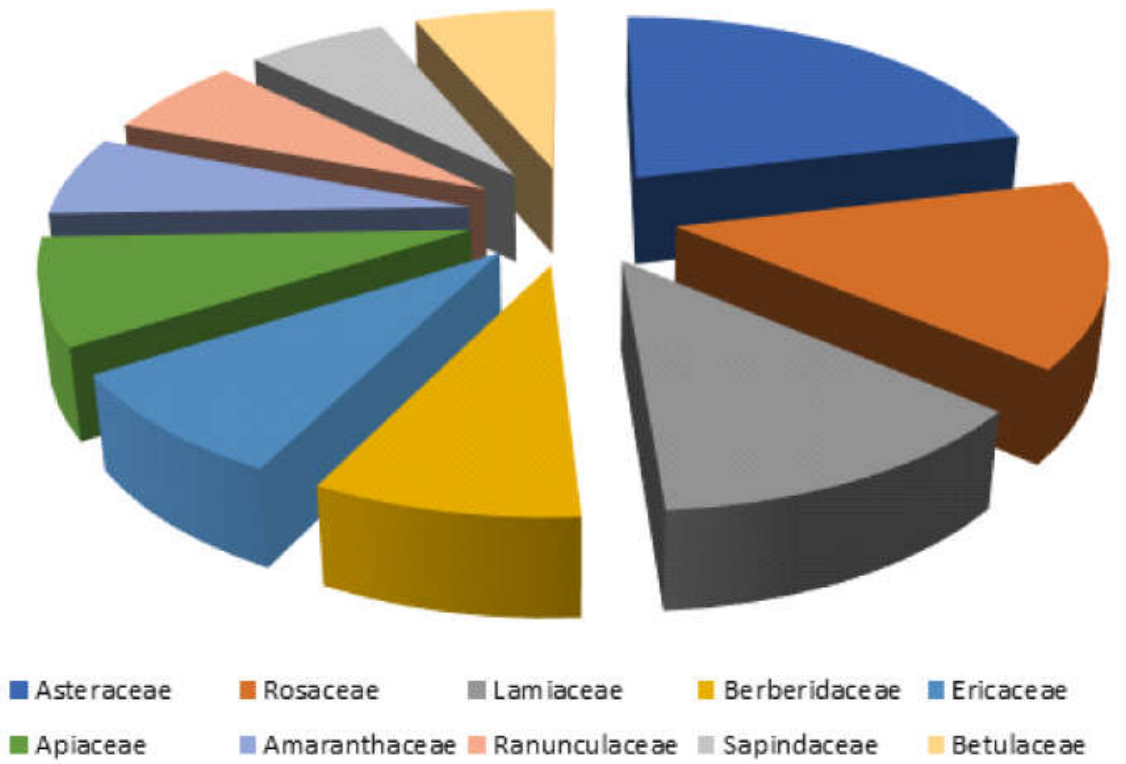

Figure 4. Contribution of ten dominant families having ethnomedicinal plants in Pindari valley

Most of the plant species are known by their own local name by the local people of the region as listed out in Table 1. Local people deliberately utilize these plants because of their high beliefs of nutrients and medicinal qualities.

\section{CONCLUSION}

From this study, it can be established that Pindari valley has a treasure of ethnomedicinal plants. The traditional knowledge about the habitat, parts used and way of using ethnomedicinal species is preserved. The exploration of the valley for discovering more valuable plants requires cumulative efforts towards attaining more knowledge about the medicinally important plants. But there should be sustainable utilization of these plants so as to conserve them from the threat of extinction.

\section{Acknowledgements}

Authors are thankful to the Director, CSIR-NBRI, Lucknow for encouragement and providing facilities to carry out the work. We also acknowledge Dr. Tariq Husain, ex-senior principal scientist, CSIR-NBRI for his consistent guidance. We also thank CSIR, New Delhi for providing Junior Research fellowship to first and second author.

\section{LITERATURE CITED}

Agnihotri, P.; Katiyar, P. \& Husain, T. 2016. Ethnobotanically important plants of Kishanpur Wildlife Sanctuary, Uttar Pradesh, India. Pleione 10(1): 32 - 42.

Aitken, S. \& Badola, H. K. 2003. The Himalayas of India: A treasury of medicinal plants under siege. Biodiversity. 4(3): 3 - 13.

Balachandhran, G. \& Arora, S. 2006. India's Third National Report to Convention on Biological Diversity: Executive Summary, Ministry of Environment and Forest, Government of India, Centre for Environment Education, Ahmedabad. India. Pp. 21.

Balik, M.J. \& Cox, P.A. 1996. Plants, People and Culture: The science of Ethnobotany. Scientific American Library, New York. Pp. 228. 
Bisht, V. K.; Rana, C. S.; Negi, J. S.; Bhandari, A. K.; Purohit, V.; Kuniyal, C. P. \& Sundriyal, R.C. 2012. Lamiaceous ethno-medico-botanicals in Uttarakhand Himalaya, India. Journal of Medicinal Plant Research 6(26): 4281 - 4291.

Dutt, B.; Nath, D.; Chauhan, N.S.; Sharma, K.R. \& Sharma, S.S. 2014. Ethno-medicinal Plant Resources of Tribal Pangi Valley in District Chamba, Himachal Pradesh, India. International Journal of Stress Management 5(3): 416 - 421.

Ford, D.; Easton, D.F.; Bishop, D.T.; Narod, S.A. \& Goldgar, D.E. 1994. Risks of cancer in BRCA1-mutation carriers. The Lancet. 343 (8899): $692-695$.

Joshi, S.; Upreti, D.K. \& Das, P. 2011. Lichen diversity assessment in Pindari Glacier Valley of Uttarakhand, India. Geophytology. 41(1-2): 25 - 41.

Kandpal, V. \& Sathyakumar, S. 2010. Distribution and relative abundance of mountain ungulates in Pindari Valley, Nanda Devi Biosphere Reserve, Uttarakhand, India. Galemys 22(no. especial): $277-294$.

Kholiya, D.; Rawat, A.; Kukreti, R. \& Joshi, P. 2018. Strategic Management for Eco-Tourism Destinations: Pindari and Roopkund areas of Nanda Devi Biosphere Reserve of Uttarakhand. Amity Research Journal of Tourism, Aviation and Hospitality. 3(1): 50 - 57.

Kumari, P.; Samant, S.S. \& Puri, S. 2018. Diversity, distribution, indigenous uses and conservation of medicinal plants in central Himachal Pradesh, North Western Himalaya. Journal of Medicinal Plants Studies 6(5): $45-68$.

Naithani, S. \& Nand, K. 2018. Ethnobotanical uses of wild medicinal plants by the local community in the Asi Ganga Sub-basin, Western Himalaya. Journal of Complementary Medicine Research. 9(1): 34.

Pala, N.A.; Negi, A.K. \& Todaria, N.P. 2010. Traditional uses of medicinal plants of Pauri Garhwal, Uttarakhand. New York Science Journal, 3(6): 61 - 65.

Pandey, A.K. \& Tripathi, Y.C. 2017. Ethnobotany and its relevance in contemporary research. Journal of Medicinal Plants Studies 5(3): 123 - 129.

Pandey, N.C.; Bhatt, D.; Arya, D.; Chopra, N.; Upreti, B.M.; Joshi G.C. \& Tewari, L.M. 2017. Diversity of ethno-medicinal plant: A case study of Bageshwar district Uttarakhand. Journal of Medicinal Plants Studies 5(2): $11-24$.

Rana, M.S. \& Samant, S.S. 2011. Diversity, indigenous uses and conservation status of medicinal plants in Manali wildlife sanctuary, North western Himalaya. Indian Journal of Traditional Knowledge 10(3): 439 - 459.

Rao, T.A. 1960, A botanical tour of Pindari Glaciers and Kumuon Hill Stations. Bulletin of the Botanical Survey of India, 2: $61-94$.

Rawat, R. \& Vashistha, D P. 2011. Common Herbal Plant in Uttarakhand, Used in the Popular Medicinal Preparation in Ayurveda. Int. J. Pharmacogn. Phytochem. 3(3): 64 - 73.

Shah, N.C.\& Joshi, M.C. 1971. An Ethnobotanical Study of the Kumaon Region of India. Econ. Bot. 25 (4): $414-422$.

Singh, K.K. \& Kumar, K. 2000. Ethnobotanical Wisdom of Gaddi Tribe in Western Himalaya. Bishen Singh Mahendra Pal Singh, Dehra Dun. Pp.148.

Siwach, M.; Siwach, P.; Solanki P. \& Gill, A. R. 2013. Biodiversity conservation of Himalayan medicinal plants in India: A retrospective analysis for a better vision. International Journal of Biodiversity Conservation 5(9): 529 - 540.

Topwal, M. \& Uniyal, S. 2018. Review on Important Ethno- Medicinal Plants in Uttarakhand. International Journal of Pure and Applied Bioscience 6(2): 455 - 464. 